\title{
SAS and SPSS macros to calculate standardized Cronbach's alpha using the upper bound of the phi coefficient for dichotomous items
}

\author{
Wei Sun, Chih-Ping Chou, Alan W. Stacy, Huiyan Ma, \\ Jennifer Unger, and Peggy Gallaher \\ University of Southern California, Los Angeles, California
}

\begin{abstract}
Cronbach's $\alpha$ is widely used in social science research to estimate the internal consistency of reliability of a measurement scale. However, when items are not strictly parallel, the Cronbach's $\alpha$ coefficient provides a lower-bound estimate of true reliability, and this estimate may be further biased downward when items are dichotomous. The estimation of standardized Cronbach's $\alpha$ for a scale with dichotomous items can be improved by using the upper bound of coefficient $\phi$. SAS and SPSS macros have been developed in this article to obtain standardized Cronbach's $\alpha$ via this method. The simulation analysis showed that Cronbach's $\alpha$ from upper-bound $\phi$ might be appropriate for estimating the real reliability when standardized Cronbach's $\alpha$ is problematic.
\end{abstract}

Reliability of the measures derived from tests and questionnaires refers to the consistency, stability, and repeatability of a data collection instrument (Crocker \& Algina, 1986; Cronbach, 1990; Traub, 1994). A reliable instrument will have consistent results if repeated over time or if used by two different investigators. Internal consistency of reliability refers to the extent to which all parts of the measurement technique are measuring the same concept. For example, when developing a questionnaire to measure implicit cognition, each question should provide a measure of implicit cognition consistent with the overall results of the test. Although multiple tests are required for estimating stability and equivalence of reliability, only a single test is needed for estimating internal consistency. Cronbach's $\alpha$ coefficient is the most widely used index to estimate the internal consistency of reliability of a scale containing multiple items.

In social science, multiple items are often used in assessments of memory, personality, and other psychological constructs. Under many circumstances, such items are combined (e.g., summed) to form a scale. The scale is then frequently subjected to some form of reliability analysis, and investigators usually report internal consistency in the form of Cronbach's $\alpha$. For a scale with $k$ items, the standardized Cronbach's coefficient $\alpha$ can be computed from a correlation matrix using the following formula (Cronbach, 1951; Nunnally, 1967):

$$
\alpha=\frac{k \bar{r}}{1+(k-1) \bar{r}},
$$

where $\bar{r}$ is the mean of the $k \cdot(k-1) / 2$ nonredundant correlation coefficients.
The standardized Cronbach's coefficient $\alpha$ is not a lower bound to the true reliability, and it may yield under- or overestimates of the true reliability (Osburn, 2000; Zimmerman, Zumbo, \& Lalonde, 1993). Theoretically, when the items of a composite measure are congeneric, $\tau$ equivalent, or essentially $\tau$ equivalent, standardized $\alpha$ will always exceed the true reliability (Sočan, 2000). However, if the measure contains a small number of heterogeneous items, the standardized Cronbach's $\alpha$ tends to underestimate the true reliability of a measure (Osburn, 2000). And if the items of a scale are characterized by multiple moderately correlated factors, the standardized Cronbach's coefficient $\alpha$ may be seriously underestimated as well. In practice, the standardized Cronbach's $\alpha$ almost always underestimates true reliability (Sočan, 2000).

The underestimation of Cronbach's $\alpha$ is more serious when the items are dichotomous, because correlations among dichotomous items ( $\phi$ coefficients) tend to underestimate true correlations (Brogden, 1946a, 1946b; Gulliksen, 1945). In this article, we propose a method to ameliorate the underestimation of standardized Cronbach's $\alpha$ by incorporating the upper bound of the $\phi$ coefficient into the calculation of standardized Cronbach's $\alpha$.

\section{Upper Bound of Correlation Coefficient $\phi$}

The correlation coefficient $\phi$ is usually calculated to reflect the relationship between two dichotomous variables. The standardized Cronbach's coefficient $\alpha$, based on the average correlation among items (see Equation 1), is computed from $\phi$ when items are dichotomous. A $2 \times 2$ frequency table (see Table 1) can be structured for two dichotomous items, $X_{i}$ and $X_{j}$, with "yes" and "no" responses.

W.Sun,wsun@usc.edu 
Table 1

A $2 \times 2$ Table for Dichotomized Items

\begin{tabular}{|c|c|c|c|c|}
\hline & & \multicolumn{2}{|c|}{$X_{j}$} & \multirow[b]{2}{*}{ Total } \\
\hline & & Yes & $\overline{\mathrm{No}}$ & \\
\hline \multirow[t]{3}{*}{$X_{i}$} & Yes & $\mathrm{a}$ & $\mathrm{b}$ & $a+b$ \\
\hline & No & $\mathrm{c}$ & $\mathrm{d}$ & $\mathrm{c}+\mathrm{d}$ \\
\hline & Total & $a+c$ & $b+d$ & $N$ \\
\hline
\end{tabular}

$\phi$ can be calculated using the following equation:

$$
\phi=\frac{a d-b c}{\sqrt{(a+c)(b+d)(a+b)(c+d)}} .
$$

The upper bound of $\phi$ between a pair of dichotomous items can be calculated using the following formula (Lord \& Novick, 1968; Shrout \& Parides, 1992):

$$
\phi_{\max }=\sqrt{\pi_{j}\left(1-\pi_{i}\right) / \pi_{i}\left(1-\pi_{j}\right)},
$$

where $\pi_{i}$ and $\pi_{j}$ are the endorsement ("yes" response) rates of the two items, with $\pi_{i} \geq \pi_{j}$.

With the upper bound of $\phi$, an estimate of the standardized Cronbach's coefficient $\alpha$ can be obtained directly from Equation 1.

\section{Introduction of SAS and SPSS Macros}

As described in this article, an SAS macro has been developed to calculate the standardized Cronbach's coefficient $\alpha$ from the upper bound of $\phi$. In this macro, the original Cronbach's coefficient $\alpha$ is obtained from dichotomous items with the SAS procedure PROC CORR. The upper bound of $\phi$ is calculated from each pair of dichotomous items using Equation 3. The standardized Cronbach's coefficient $\alpha$ is obtained directly from the mean of the upper-bound $\phi s$.

The SAS macro is provided in Appendix A. In this macro, there are three major input variables defined. One is for the name of the input data set, another is for the string of dichotomous variables, and the third is for the name of the output data set in which the original standardized Cronbach's $\alpha$ and standardized Cronbach's $\alpha$ using upper-bound $\phi$ will be stored. A data set for testing purposes is attached in Appendix B. An example of SAS codes to run the macro, as well as the SAS output, is provided in Appendix C.

The corresponding SPSS version of our code (syntax and macros) to facilitate calculation of standardized Cronbach's $\alpha$ using upper-bound $\phi$ was developed also. The code for both the syntax and the macros is provided in Appendix D. The SPSS statements to input the same test data set, the syntax as the SPSS macros run, and the output are attached in Appendix E.

\section{Simulation Analysis}

The Alcohol Expectancy Questionnaire is an established instrument used in alcohol studies (e.g., Leigh \& Stacy, 1993, 2004; MacKinnon, Nohre, Pentz, \& Stacy, 2000). There are 19 items on the alcohol expectancy scale. All items have four categories as potential answers. Positive outcome expectancy items from the scale reported in
Leigh and Stacy (1993) were factor analyzed in a sample of 1,107 adolescents. The three highest-loading items were chosen to form a pseudo-instrument used in the simulation analysis.

The correlations among these three picked items were $.75, .57$, and .60 . Using the correlation matrix, a Monte Carlo study was carried out to determine the percentage of under- or overestimation of the simulated dichotomous items in comparison with the real reliability. First, from the correlation matrix of the three picked items, the continuous data with three variables in normal distribution were simulated. The Cronbach's $\alpha$ from these simulated continuous variables was considered the real reliability (.843). Second, the dichotomous variables were derived from the continuous data with the defined endorsement rates. Then the standardized Cronbach's $\alpha$ and Cronbach's $\alpha$ from upper-bound $\phi$ were obtained from the dichotomized three items with the SAS macro developed in this study. For each endorsement rate, 100 samples generated, 300 cases in each sample. Table 2 presents the results of this simulation analysis.

This table shows that in the simulated conditions, standardized Cronbach's $\alpha$ seriously underestimated the real reliability, and Cronbach's $\alpha$ from upper-bound $\phi$ was closer to the true reliability. However, the table also shows that Cronbach's $\alpha$ from upper-bound $\phi$ was an overly optimistic estimation of reliability (intermediate endorsement rate, $7.8 \%$; high endorsement rate, $0.1 \%$ ), whereas standardized Cronbach's $\alpha$ underestimated the reliability (intermediate endorsement rate, $-17.6 \%$; high endorsement rate, $-23.7 \%)$. We concluded that Cronbach's $\alpha$ from upper-bound $\phi$ might be useful in evaluating the reliability of a scale in conditions in which standardized Cronbach's $\alpha$ is problematic. To clearly reveal the relation between true reliability and Cronbach's $\alpha$ from upperbound $\phi$, however, a more extensive simulation study is needed.

\section{AUTHOR NOTE}

Correspondence relating to this article may be sent to W. Sun, $1000 \mathrm{~S}$. Fremont, Unit 8, Alhambra, CA 91803 (e-mail: wsun@usc.edu).

\section{REFERENCES}

BRogden, H. E. (1946a). The effect of bias due to difficulty factors in product-moment item intercorrelations on the accuracy of estimation of reliability by the Kuder-Richardson formula Number 20. Educational \& Psychological Measurement, 6, 517-520.

BRogDEn, H. E. (1946b). Variation in test validity with variation in the distribution of item difficulties, number of items, and degree of their intercorrelation. Psychometrika, 11, 197-214.

Crocker, L., \& Algina, J. (1986). Introduction to classical and modern test theory. New York: Holt, Rinehart \& Winston.

Cronbach, L. J. (1951). Coefficient alpha and the internal structure of tests. Psychometrika, 16, 297-334.

Cronbach, L. J. (1990). Essentials of psychological testing (5th ed.). New York: HarperCollins.

GULLIKSEN, H. (1945). The relation of item difficulty and inter-item correlation to test variance and reliability. Psychometrika, 10, 79-91.

Leigh, B. C., \& Stacy, A. W. (1993). Alcohol outcome expectancies: Scale construction and predictive utility in higher order confirmatory models. Psychological Assessment, 5, 216-229.

LeIGH, B. C., \& StACY, A. W. (2004). Alcohol expectancies and drinking in different age groups. Addiction, 99, 215-227. 
Table 2

Comparison of Standardized Cronbach's $\alpha$ and Cronbach's $\alpha$ From Upper-Bound $\phi$ on Simulated Dichotomous Items From a Pseudo Three-Item Alcohol Expectancy Instrument

\begin{tabular}{|c|c|c|c|c|c|c|}
\hline \multirow[b]{2}{*}{ Endorsement Rated } & \multicolumn{3}{|c|}{ Standardized Cronbach's $\alpha$} & \multicolumn{3}{|c|}{$\begin{array}{c}\text { Cronbach's } \alpha \text { From } \\
\text { Upper-Bound } \phi\end{array}$} \\
\hline & Mean ${ }^{a}$ & $S D^{\mathrm{b}}$ & Percentc & Mean ${ }^{\mathrm{a}}$ & $S D^{\mathrm{b}}$ & Percentc \\
\hline Low & .641 & .044 & -23.9 & .842 & .023 & -0.1 \\
\hline Intermediate & .694 & .033 & -17.6 & .908 & .013 & 7.8 \\
\hline High & .643 & .044 & -23.7 & .844 & .022 & 0.1 \\
\hline Low \& high & .480 & .036 & -43.1 & .575 & .025 & -31.8 \\
\hline Low \& intermediate & .611 & .041 & -27.5 & .746 & .022 & -11.4 \\
\hline Intermediate $\&$ high & .598 & .038 & -29.0 & .713 & .022 & -15.4 \\
\hline
\end{tabular}

Note- - Mean of standardized Cronbach's $\alpha$ over 100 replications in the simulation analysis. bSD of standardized Cronbach's $\alpha$ over 100 replications in the simulation analysis. 'Percent of Cronbach's $\alpha$ from dichotomous items under-/overestimated on the Cronbach's $\alpha$ from continuous items. The Cronbach's $\alpha$ from simulated continuous data is equal to .843 . dEndorsement rate in the derived dichotomous items. These rates were as follows: low, .1, .2, .3; intermediate, $.4, .5, .6$; high, .7, .8, .9; low \& high, $.1, .2, .9$; low \& intermediate, $.1, .2, .5$; intermediate \& high, $.4, .8, .9$.

LoRD, F. M., \& Novick, M. R. (1968). Statistical theories of mental test scores. Reading, MA: Addison-Wesley.

MacKinnon, D. P., Nohre, L., Pentz, M. A., \& Stacy, A. W. (2000). The alcohol warning and adolescents: 5 -year effects. American Journal of Public Health, 90, 1589-1594.

Nunnally, J. C. (1967). Psychometric theory. New York: McGraw-Hill.

Osburn, H. G. (2000). Coefficient alpha and related internal consistency reliability coefficients. Psychological Methods, 5, 343-355.

Shrout, P. E., \& Parides, M. (1992). Conventional factor analysis as an approximation to latent trait models for dichotomous data. International Journal of Methods in Psychiatric Research, 2, 55-65.

Sočan, G. (2000). Assessment of reliability when test items are not essentially $\tau$-equivalent. Developments in Survey Methodology, 15, 23-35.

Traub, R. E. (1994). Reliability for the social sciences: Theory and applications. Thousand Oaks, CA: Sage.

Zimmerman, D. W., Zumbo, B. D., \& Lalonde, C. (1993). Coefficient alpha as an estimate of test reliability under violation of two assumptions. Educational \& Psychological Measurement, 53, 33-49.

\section{APPENDIXA \\ SAS Macro for Cronbach's Coefficient $\alpha$ From the Upper Bound of Coefficient $\phi$}

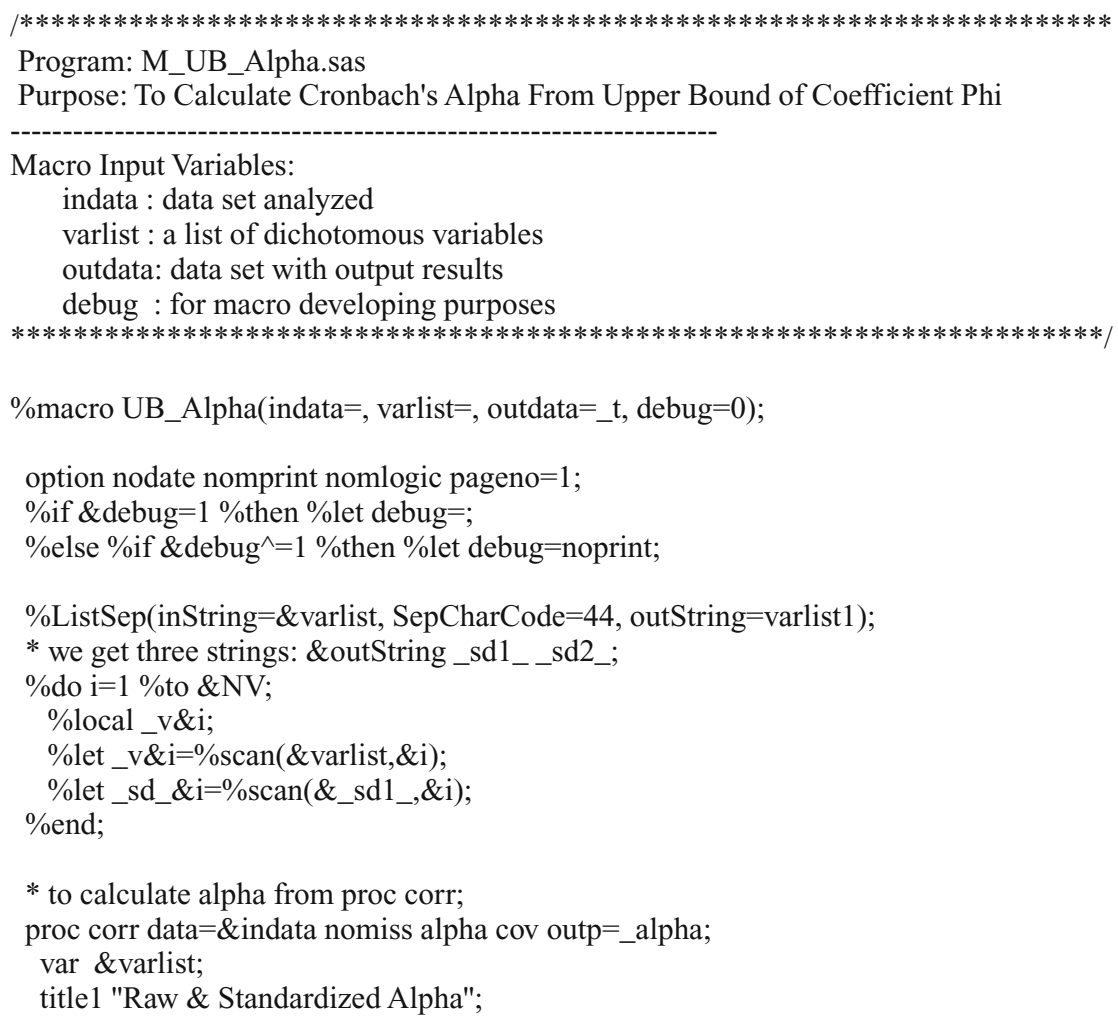

* to calculate alpha from proc corr;

proc corr data=\&indata nomiss alpha cov outp=_alpha;

var \&varlist;

title1 "Raw \& Standardized Alpha"; 
title2 "(from Proc Corr)";

run;

* to get the cov \& corr matrix;

data_corrout;

* to get the $\operatorname{cov} \&$ corr matrix;

data_corrout;

set_alpha;

if _TYPE_in ('COV', 'MEAN', 'STD', 'N', 'CORR') then output_corrout;

if_type_='RAWALPHA' then call symput('RAWALPHA', \&_v1);

if_type_='STDALPHA' then call symput('STDALPHA', \&_v1);

run;

proc sql noprint;

select \&varlist1 into: \&_sd2_

from_corrout

where_type_='STD';

quit;

*-- to calculate Cronbach's alpha upper bound ;

data_null_;

set_corrout;

if_type_="MEAN" then do;

\%do $\mathrm{i}=1$ \%to \&nv;

_p\&i=\&\&\&_v\&i;

\%end;

\%do $i=1 \%$ to \&nv;

$\%$ do $j=1 \%$ to \&nv;

$\%$ if $\& \mathrm{i}^{\wedge}=\& \mathrm{j} \%$ then $\%$ do;

if_p\&i $>=\_p \& j$ then do;

ratio_\&i._\&j=(_p\&j*(1-_p\&i))/(_p\&i*(1-_p\&j));

end;

else do;

ratio_\&i._\&j=(_p\&i*(1-_p\&j))/(_p\&j*(1-_p\&i));

end;

phi_up_\&i._\&j=sqrt(ratio_\&i._\&j);

call symput("phi_up_\&i._\&j",phi_up_\&i._\&j); \%end;

$\%$ end;

$\%$ end;

end;

run;

data_corrout2;

set_corrout;

if_type_="CORR" then do;

\%do $\mathrm{i}=1 \%$ to \&nv;

if_name_="\&\&\&_v\&i" then do;

\%do $j=1 \%$ to \&nv;

$\%$ if $\& \mathrm{i}^{\wedge}=\& \mathrm{j} \%$ then $\%$ do;

$\& \& \&$ \&\&j=\&\&\&phi_up_\&i.\&j;

$\%$ end;

$\%$ end;

end;

\%end;

end;

if_type_="COV" then do;

\%do i=1 \%to \&nv;

if_name_="\&\&\&_v\&i" then do;

\%do $\mathrm{j}=1 \%$ to \&nv;

\%if $\& \mathrm{i}^{\wedge}=\& \mathrm{j} \%$ then \%do;

sd1=symget("_sd_\&\&\&_v\&i"); 


\section{APPENDIX A (Continued)}

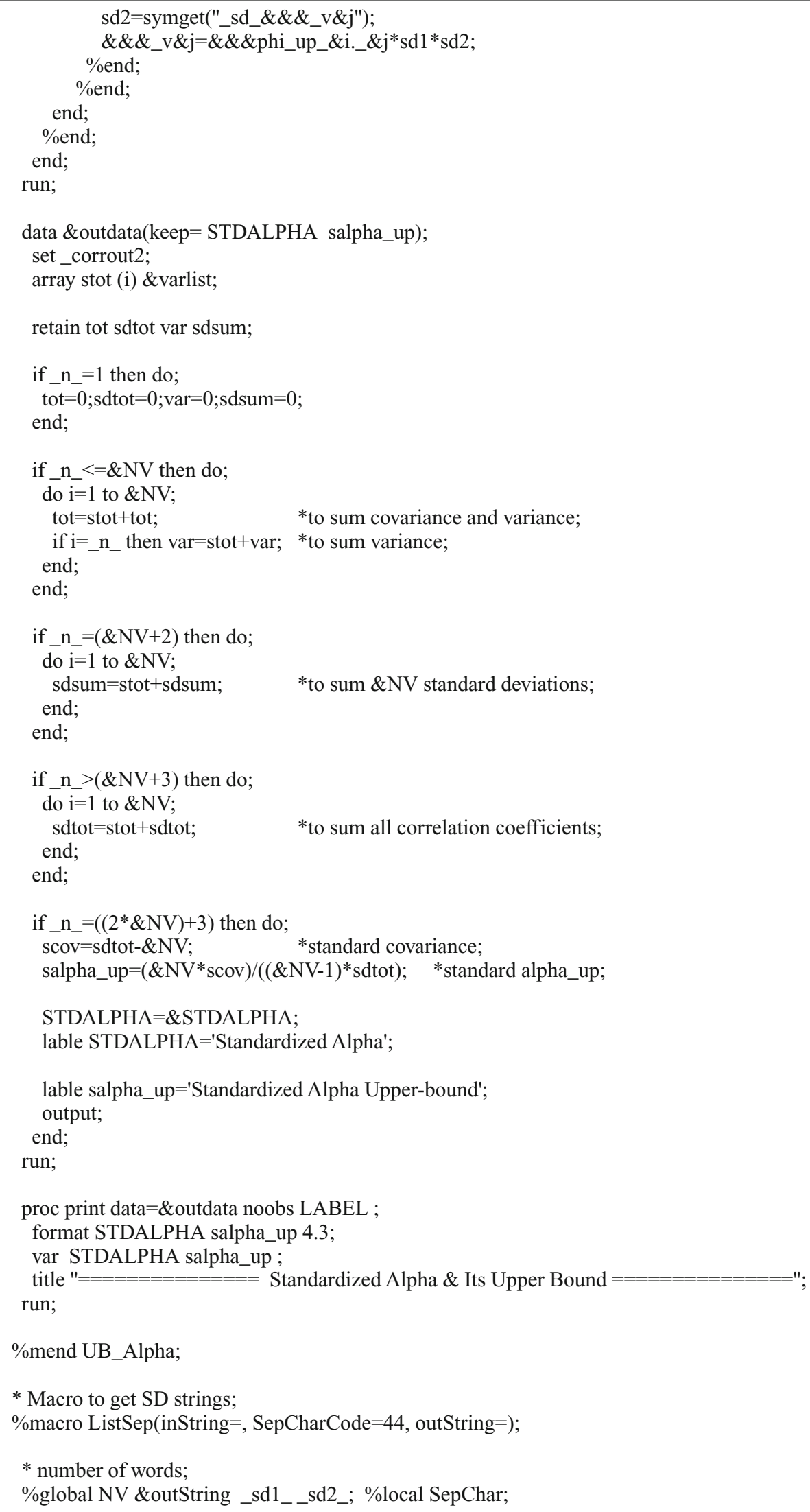




\section{APPENDIX A (Continued)}

data_null_; array_a \&inString ;

call symput('NV',left(dim(_a)));

run;

\%*put \&inString; \%let_outString=; \%*put \&SepChar;

\%do i=1 \%to \&NV;

$\%$ let $\mathrm{t}=\%$ scan $(\&$ inString,\&i);

\%if \&i=1 \%then \%do;

$\%$ let \&outString=\&t;

$\%$ let_sd1_=_sd_\&t;

$\%$ let_sd2_=_sd_\&t;

$\%$ end;

$\%$ else \%if \&i>1\%then \%do;

$\%$ let SepChar=\%sysfunc(byte(\&SepCharCode));

\%let \&outString=\&\&\&outString \&SepChar \&t;

$\%$ let_sd1_=\&_sd1__sd_\&t;

$\%$ let_sd2_=\&_sd2_\&SepChar :_sd_\&t;

$\%$ end;

\%end;

\%*put \&\&outString;

$\% *$ put \&_sd1_;

$\%$ *put \&_sd 2_;

\%mend ListSep;

\begin{tabular}{|c|c|c|}
\hline \multicolumn{3}{|c|}{$\begin{array}{c}\text { APPENDIX B } \\
\text { Sample of SAS Data Set }\end{array}$} \\
\hline \multicolumn{3}{|c|}{$\begin{array}{l}\text { data t; } \\
\text { input v1 v2 v3; } \\
\text { datalines; }\end{array}$} \\
\hline 1 & 1 & 1 \\
\hline 1 & 0 & 0 \\
\hline 1 & 1 & 0 \\
\hline 1 & 1 & 1 \\
\hline 1 & 0 & 1 \\
\hline 1 & 0 & 1 \\
\hline 1 & 1 & 1 \\
\hline 1 & 0 & 0 \\
\hline 0 & 1 & 0 \\
\hline 1 & 1 & 1 \\
\hline 1 & 1 & 1 \\
\hline 1 & 1 & 0 \\
\hline 1 & 1 & 1 \\
\hline 1 & 1 & 1 \\
\hline 0 & 1 & 0 \\
\hline 1 & 1 & 1 \\
\hline 0 & 1 & 0 \\
\hline 1 & 1 & 1 \\
\hline 1 & 1 & 1 \\
\hline 1 & 1 & 1 \\
\hline 1 & 1 & 0 \\
\hline 1 & 1 & 1 \\
\hline 1 & 1 & 1 \\
\hline 1 & 1 & 1 \\
\hline 1 & 0 & 1 \\
\hline 1 & 0 & 0 \\
\hline 1 & 1 & 1 \\
\hline 0 & 0 & 0 \\
\hline 1 & 1 & 1 \\
\hline 1 & 1 & 1 \\
\hline $\begin{array}{l}; \\
\text { run }\end{array}$ & & \\
\hline
\end{tabular}




\section{APPENDIX C}

Sample of Using the SAS Macro and of Its Output

\section{Sample of Using the SAS Macro}

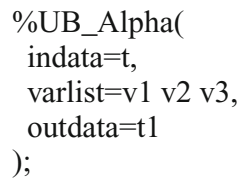

\begin{tabular}{cc}
$\begin{array}{c}\text { Standardized } \\
\text { Alpha }\end{array}$ & $\begin{array}{c}\text { Alpha } \\
\text { Upper-bound }\end{array}$ \\
0.542 & 0.865 \\
\hline
\end{tabular}

\section{APPENDIX D}

SPSS Syntax and Macros for Cronbach's Coefficient $\boldsymbol{\alpha}$ From the Upper Bound of Coefficient $\phi$

*Note: 1) The name of input data set should be $t 1$.

* 2) All dichotomous variables should be defined as v plus a number, such as v1, v2, v3, etc.

* 3) The global macro, varNumber, should be assigned an integer.

* 4) The folder, c:Itemp, exists.

SET PRINTBACK $=$ off MPRINT $=$ off .

DATA LIST LIST /c.

BEGIN DATA

1

END DATA.

save outfile 'c: |templc1.sav'.

DEFINE mp(nbvar=! tokens(1))

* macro 1

!do ! cnt $=1$ ! to !nbvar

! if $($ !cnt $=1)$ ! then

write outfile 'c:|temp \temp.sps' /"*to get percent."/"aggregate outfile 'c:|templt2.sav"'/" /break = const"/!quote(! concat('/pv',! cnt,'=mean $\left.\left.\left(\mathrm{v}^{\prime}, \text { !cnt, }\right)^{\prime}\right)\right)$.

!ifend.

!if $($ ! cnt = !nbvar $)$ !then

write outfile 'c:ltempltemp.sps' /!quote(!concat(' /pv',!cnt,'=mean(v',!cnt,').')).

!ifend.

!if (! cnt $<>1 \&$ ! cnt $<>$ !nbvar) ! then

write outfile 'c: Itemp\temp.sps' /!quote(!concat(' /pv',! cnt,'=mean(v',!cnt,')')).

!ifend.

!doend

exe.

!ENDDEFINE.

* macro 2

DEFINE upb_phi(var1=!tokens(1)/ $\operatorname{var} 2=$ ! tokens $(1) /$

do if (!var1 gt ! var2). $\operatorname{var} 3=$ ! tokens $(1))$.

+ compute !var3=sqrt(!var2*(1-!var1)/(!var1*(1-!var2)))

else if (!var1 le !var2).

+ compute !var3=sqrt(!var1*(1-!var2)/(!var2*(1-!var1))).

end if.

!ENDDEFINE. 


\section{APPENDIX D (Continued)}

* macro 3 --------------- .

DEFINE upb_phi1(nbvar=!tokens(1))

!let ! const $1=1$.

!do !cnt=1 !to !nbvar

!let !cnt1 = !length(!concat(!blanks(!cnt), !blanks(!const1))).

!do !cntt=!cnt1 ! to !nbvar

!let !vt1 $=$ !concat('pv',! cnt).

!let ! vt2 =! concat('pv',! centt).

!let !vt3 $=$ ! concat('pv',! cnt,',',! cntt).

write outfile 'c:|temp \temp.sps' /!quote(!concat('upb_phi var1=',!vt1, 'var2=',!vt2, ' var3=',,!vt3,'.')).

!doend

!doend

exe.

!ENDDEFINE.

* macro 4 ------------

DEFINE M_alphaUP(nbvar=!tokens(1))

!let ! ttt $=$ ! concat(' ').

!do !cnt $=1$ ! to !nbvar

!do ! cnt $1=2$ ! to !nbvar

!if $($ !cnt $=1 \&$ ! cnt $1=2)$ ! then

!let ! $\mathrm{ttt}=$ ! concat $\left({ }^{\prime} 0^{\prime}\right)$

!ifend

!if (!cnt $<$ !cnt1) !then

!let ! ttt = !concat (!ttt,'+pv',!cnt,',',!cnt1).

!ifend

!doend

!doend

compute totr $=$ !ttt.

compute totn=!nbvar*(!nbvar-1)/2.

compute avgr=totr/totn.

compute salpha_up=!nbvar*avgr/(1+(!nbvar-1)*avgr $)$.

exe.

!ENDDEFINE.

* macro 5 -------------- .

DEFINE Mcorr(nbvar=! tokens(1))

!let !ttt= !concat('v', !nbvar)

reliability variables $=\mathrm{v} 1$ to $! \mathrm{ttt}$

/model=alpha

/matrix=out ('c:|templcorr.sav') noprint.

\section{!ENDDEFINE.}

* macro 6 ----------- .

DEFINE Msum(nbvar=!tokens(1))

!do ! cnt $=1$ ! to !nbvar

!if (!cnt =1) !then

write outfile 'c: Itemp \temp.sps' /"*to get percent."/"aggregate outfile 'c:ltemp $\backslash$ t2.sav"'/" /break = const"/!quote(!concat(' /sv',!cnt,'=sum( $\left(v^{\prime}\right.$, !cnt,')')).

!ifend.

! if (!cnt $=$ !nbvar) !then

write outfile 'c:|temp \temp.sps' /!quote(!concat(' /sv',! cnt,'=sum(v',!cnt,').')).

write outfile 'c: Itemp temp.sps' /!quote(! concat(' /n=sum(const).')).

!ifend.

!if (!cnt $<1 \&$ ! cnt $<>$ !nbvar $)$ !then 


\section{APPENDIX D (Continued)}

write outfile 'c:|temp\temp.sps' /!quote(! concat(' /sv',!cnt,'=sum( $\left(v^{\prime}\right.$, !cnt,')')).

!ifend.

!doend

exe.

!ENDDEFINE.

* macro 7 ---------

DEFINE Malpha(nbvar=!tokens(1))

!let ! $\mathrm{ttt}=$ ! concat('sv1').

!do !cnt $=2$ ! to !nbvar

!let ! ttt $=$ !concat $\left(! \mathrm{ttt},{ }^{\prime}+\mathrm{sv}^{\prime}\right.$, ! cnt $)$.

!doend

compute totr $=(! \mathrm{ttt}-! \mathrm{nbvar}) / 2$.

compute totn=! nbvar* $($ !nbvar-1) $/ 2$.

compute avgr=totr/totn

compute salpha $=$ !nbvar*avgr/(1+(!nbvar-1)*avgr).

!ENDDEFINE.

get file 'c:|templc1.sav'.

$\mathrm{mp}$ nbvar=varnumber.

get file='c: |temp $\backslash$ t 1. sav'.

compute const $=1$.

execute.

*list.

include 'c: \temp \temp.sps'.

get file 'c: ttemp $\backslash c 1$. sav' $^{\prime}$

upb_phi1 nbvar=varnumber.

get file 'c: $\mid$ temp $\backslash$ t2.sav'.

include 'c: Itempltemp.sps'.

save outfile 'c:|temp\salpha_phi.sav'.

*list.

get file 'c: ttemp $\backslash$ salpha_phi.sav'.

M_alphaUP nbvar=varnumber.

save outfile 'c:|temp\salpha_phi.sav'

/keep salpha_up.

*list.

get file 'c: |temp $\backslash t 1 . s a v '$ '.

Mcorr nbvar=varnumber

get file 'c: Itemplcorr.sav'. select if (rowtype_='CORR').

save outfile 'c:|templcorr1.sav'

/drop rowtype_varname_.

get file 'c: Itemp $\backslash$ corr 1.sav'. compute const $=1$.

execute.

*list.

get file 'c: \temp $\backslash \mathrm{c} 1 . \mathrm{sav}$ '.

Msum nbvar=varnumber.

get file 'c: |templcorr1.sav'.

compute const $=1$.

execute.

$*$ list. 


\section{APPENDIX D (Continued)}

include 'c: |temp $\backslash$ temp.sps'

get file 'c: \temp $\backslash$ t2.sav'.

Malpha nbvar=varnumber.

save outfile 'c: Itemp\salpha.sav'

/keep salpha.

*list.

match files file='c:|temp $\backslash$ salpha_phi.sav' /file='c: temp $\backslash$ salpha.sav'.

variable labels

salpha_up "Standardized Cronbach's alpha from Upper Bound phi Coefficient" salpha "Standardized Cronbach's alpha".

save outfile 'c: |temp\alpha.sav'.

set header $=$ no printback $=$ no.

formats salpha_up(F5.3) salpha(F5.3).

variable labels

salpha_up "Cronbach's alpha from UB phi"

salpha "Standardized Cronbach's alpha".

display labels.

list.

erase file $={ }^{\prime} \mathrm{c}: \mid$ temp $\backslash \mathrm{t} 2 . \mathrm{sav}^{\prime}$.

erase file $=$ 'c: $\mid$ temp $\backslash$ corr.sav'.

erase file $={ }^{\prime} \mathrm{c}: \mid$ temp $\backslash$ temp. .sps ,

erase file $=$ 'c: $\mid$ temp $\backslash$ salpha_phi.sav'.

erase file $=$ 'c: $\mid$ temp $\backslash$ salpha.sav '

erase file $=$ 'c: $\mid$ temp $\backslash$ corr 1 .sav

erase file $=$ 'c: $\mid$ temp $\backslash$ c1.sav

APPENDIX E

Test of SPSS Syntax and Macros

1. Data for Test

DATA LIST LIST / v1 v2 v3 .

BEGIN DATA

$\begin{array}{lll}1 & 1 & 1 \\ 1 & 0 & 0 \\ 1 & 1 & 0 \\ 1 & 1 & 1 \\ 1 & 0 & 1 \\ 1 & 0 & 1 \\ 1 & 1 & 1 \\ 1 & 0 & 0 \\ 0 & 1 & 0 \\ 1 & 1 & 1 \\ 1 & 1 & 1 \\ 1 & 1 & 0 \\ 1 & 1 & 1 \\ 1 & 1 & 1 \\ 0 & 1 & 0 \\ 1 & 1 & 1 \\ 0 & 1 & 0 \\ 1 & 1 & 1 \\ 1 & 1 & 1 \\ 1 & 1 & 1\end{array}$


APPENDIX E (Continued)

$\begin{array}{lll}1 & 1 & 0 \\ 1 & 1 & 1 \\ 1 & 1 & 1 \\ 1 & 1 & 1 \\ 1 & 0 & 1 \\ 1 & 0 & 0 \\ 1 & 1 & 1 \\ 0 & 0 & 0 \\ 1 & 1 & 1 \\ 1 & 1 & 1\end{array}$

END DATA.

save outfile 'c: |temp $\backslash t 1$. sav'.

* to DEFINE the number of variables.

DEFINE varNumber()

3

!ENDDEFINE.

2. Information for Running the Macros

* a) The path needs to be changed if necessary.

* b) The syntax and macros in Appendix D need to be saved in mUB_Alpha.sps. include 'c: Itemp $\backslash \mathrm{mUB}$ _Alpha.sps'.

\section{Output}

List of variables on the working file

salpha_up (1) Cronbach's alpha from UB phi

salpha (2) Standardized Cronbach's alpha

salpha_up salpha

$.865 \quad .542$

(Manuscript received May 31, 2004;

revision accepted for publication September 17, 2005.) 